\title{
Comparison of Immunogenetic Properties of Glial Tumors with Advanced Magnetic Resonance Imaging Findings
}

\author{
Burak GEZER ${ }^{1}$, Ali MUTLUKAN²${ }^{2}$, Mert SAHINOGLU ${ }^{3}$, PInar KARABAGLI ${ }^{4}$, Ender KOKTEKIR ${ }^{3}$, \\ Hakan KARABAGLI ${ }^{3}$ \\ ${ }^{1}$ Tatvan State Hospital, Department of Neurosurgery, Bitlis, Turkey \\ ${ }^{2}$ Serik State Hospital, Department of Neurosurgery, Antalya, Turkey \\ ${ }^{3}$ Selcuk University, Faculty of Medicine, Department of Neurosurgery, Konya, Turkey \\ ${ }^{4}$ Selcuk University, Faculty of Medicine, Department of Pathology, Konya, Turkey \\ This study has been presented at the $28^{\text {th }}$ Annual Scientific Congress of Turkish Neurosurgery Society between 4 and 8 April 2018 at \\ Antalya, Turkey
}

Corresponding author: Ender KOKTEKIR enderkoktekirnrs@hotmail.com

\section{ABSTRACT}

AIM: To investigate the immunogenetic properties of glial tumors according to the World Health Organization 2016 glial tumor classification and develop an accurate diagnosis and treatment strategy by comparing preoperative advanced magnetic resonance (aMRI) technique findings of these results.

MATERIAL and METHODS: This study was conducted at the Department of Neurosurgery at the Medical Faculty Hospital of Selcuk University between January 1, 2010 and January 4, 2017 and included 50 patients. MR spectroscopy (MRS), MR perfusion (PWI), and MR diffusion (DWI) were performed in 50 patients preoperatively. Patient data were obtained from the hospital's information system. Pathological diagnosis of all patients was taken from the department of pathology at the same medical faculty.

RESULTS: Among the patients included in the study, 11 were grade II (22\%), 7 were grade III (14\%), and 32 were grade IV (64\%). All patients were IDH1 stained: 22 were IDH mutant (44\%) and 28 were IDH wild-type (56\%). A statistically significant difference in the survival time was observed between IDH mutant and wild-type. IDH-mutant tumors were commonly located in the frontal lobe and IDH wild-type tumors in the parietal and temporal lobes. A significant difference in PWI relative cerebral blood volume ( $\mathrm{rCBV}$ ) and DWI apparent diffusion coefficients (ADC) was noted among grade II, III, and IV tumor groups. The PWI rCBV cut-off value for grade IV tumors was 2.05 (90\% sensitivity, 78\% specificity). No difference in the Cho/Cr ratio among grade II, III, and IV tumor groups was noted. A significant difference was noted between the IDH mutant and wild type in terms of PWI rCBV. The PWI rCBV cut-off value of IDH mutant-wild type was 2.15. No difference in the Cho/Cr, Cho/NAA, and DWI apparent diffusion coefficients ( $p>0.05$ ) was noted between the IDH mutant and wild type.

CONCLUSION: PWI rCBV is the most important prognostic value of aMRI used in tumor grading. PWI rCBV values could be significant in distinguishing IDH wild and mutant.

KEYWORDS: Isocitratedehydrogenase mutation, Magnetic resonance spectroscopy, Magnetic resonance perfusion, Magnetic resonance diffusion

ABBREVIATIONS: ADC: Apperent diffusion coefficient, c MRI: Conventional magnetic resonance imaging, Cho: Cholin, WHO: World Health Organization, DWI: Diffusion-weighted imaging, HGG: High grade gliom, IDH: Isocitratedehydrogenase, LGG: Low grade gliom, MR: Magnetic resonance imaging, MRS: MR spectroscopy, NAA: N-acetyl aspartate, PWI: Perfusionweighted imaging, VEGF: Vascular endothelial growth factor

Burak GEZER : $:$ :0000-0003-4881-8189

Ali MUTLUKAN : : 0000-0001-6431-0989

Mert SAHINOGLU (10): 0000-0003-0633-8304
PInar KARABAGLI (D): 0000-0002-5558-0175

Ender KOKTEKIR (1): 0000-0002-6442-6663

Hakan KARABAGLI (D) : 0000-0002-1184-3965 


\section{INTRODUCTION}

$\mathrm{G}$ lioblastomas are grade IV tumors, including de novo primary (95\%) and secondary glioblastomas that progress from clinically distinguishable grade II or III tumors. Based on the histopathological criteria, it is impossible to differentiate secondary glioblastomas from primary glioblastomas. In secondary glioblastomas ( $>70 \%)$, and rarely in primary glioblastomas $(<5 \%)$, isocitratedehydrogenase genes often contain activating mutations in IDH1 and IDH2 (the "IDH mutation" refers to both mutations). In particular, the two subtypes of the World Health Organization (WHO) grade II or III gliomas usually have IDH mutations. Gliomas with IDH mutations are clinically and genetically different from wildtype gliomas $(26,36)$.

Although the classification of gliomas based on histological characteristics of the resected tissue during operation may be useful in grading the disease and developing more targeted therapy, this strategy may be prone to bias due to sampling error and relatively subjective criteria during tumor resection. This uncertainty may lead to a series of difficult diagnoses, including mixed features of both astrocytomas and oligodendrogliomas. In 2016, the WHO glioma classification scheme was restructured to reflect the common molecular change in gliomas $(11,20,27,35)$.

Conventional magnetic resonance imaging (cMRI) has been established for the characterization of cerebral tumors and has been found useful, but its sensitivity for grading of gliomas varies between $55 \%$ and $83 \%$. MR spectroscopy (MRS) is a noninvasive method that allows various metabolites, such as choline-containing compounds (Cho), creatine and creatine phosphate (Cr), N-acetylaspartate (NAA), and pathological lactate levels, to be measured in vivo. Increased Cho and decreased NAA levels are common findings in brain tumors, and certain lactate and lipid levels can be detected. Relative cerebral blood volume ( $\mathrm{rCBV}$ ) measurements, derived from $\mathrm{PWI}$, are higher in high-grade tumors than in low-grade tumors and correlate with glioma vascularity $(3,5)$. A common measurement from diffusion-weighted imaging (DWI) is the apparent diffusion coefficient (ADC). ADC is an estimate of the magnitude of water molecule diffusion in the tissue, and a strong negative correlation between $A D C$ and tumor cellularity in gliomas has been observed $(10,14)$. As tumor grade increases, tumor vascularity, cellularity (2), and Cho/ NAA ratios also increase according to some studies.

Carrillo et al. suggested that imaging properties can be used to estimate the IDH1 mutation with $97.5 \%$ accuracy (7). In this study, no contrast was noted in all IDH1 mutant tumors, and most of the IDH1 tumors were located in the frontal lobe. Since increased vascular endothelial growth factor (VEGF) levels are associated with increased vascular permeability and contrast enhancement, the presence of expanding nonincubating tumor sites in IDH1 mutants indicates that this molecular genotype has low VEGF. Low VEGF lowers the PWI rCBV value in IDH-mutant tumors than in IDH wild-type tumors. Moreover, as a result of low vascularity, it is expected that cellularity and Cho/NAA values will be higher in IDH wild-type ones. A few studies tried to demonstrate how a relationship between IDH and advanced MR (aMRI) techniques might exist, but not enough information can support this. This study was conducted to investigate such relationship.

The aim of this study was to investigate the immunogenetic properties of glial tumors based on the WHO 2016 glial tumor classification and develop an accurate diagnosis and treatment strategy by comparing preoperative aMRI findings of these results.

\section{MATERIAL and METHODS}

This prospective study was discussed at the ethics committee of noninvasive clinical trials dated January 17, 2017. The board was approved by the Ethics Committee of Selcuk University Medical Faculty (decision number 2017/01).

This study was conducted at the Department of Neurosurgery at the Medical Faculty Hospital of Selcuk University between January 1, 2010 and January 4, 2017 and included 50 patients. MRS, MR perfusion, and MR diffusion were performed in 50 patients preoperatively. The age, gender, and pathological diagnosis of the patients were obtained from the information system of the hospital. Pathological diagnosis of all patients was taken from the Medicine of Selcuk Medical Faculty.

MRS examinations were performed at the MRI unit of Hospital Radiology Department with SIEMENS-AERA 1.5T MRI. In the MRS examination, the values of Cho/NAA and Cho/Cre were automatically recorded through the MR computer unit and recorded in PDF.

Perfusion MRI measurements were performed on perfusion maps in the solid section of the lesion, the region where the perfusion was highest, and in the other cerebral hemisphere; the white matter in the symmetry of this region was placed in the basal ganglia of the cerebral hemisphere for the lesions in the localization of the region of interest (ROI). The rCBV ratio was obtained by dividing the highest $\mathrm{ROI}$ from the lesion by $\mathrm{ROI}$ from the normal white matter in the opposite hemisphere.

In MR diffusion, the solid component of the tumor was based on the ADC map view from the cystic space and into the vein; $\mathrm{ROI}$ areas were placed. The ADC coefficient values were evaluated as Siemens $\times 10^{-3} \times 10^{-3} \mathrm{~mm}^{2} / \mathrm{s}$ using the Siemens Syngo E11.

Blocks of the best-representing preparations were determined in each case and a 4-micron thickness was cut with a Leica RM2255 microtome device. IDH1 immunohistochemistry was performed in the semiautomatic VentanaBenchMark XT immunohistochemistry-staining device. The staining procedure and antibody properties are shown in Table I. The stained preparations were evaluated by two pathologists using the Olympus BX53, double-headed light microscope.

In the immunohistochemical staining with IDH1 antibody, two cases, previously known to be positive and negative, were used as positive and negative control groups, respectively. In both mutations, tumor areas were evaluated by marking the preparations. 


\section{Statistical Analysis}

SPSS for Windows 18.0 program was used for statistical analysis. The results were evaluated with a confidence interval of $95 \%$ and a significance level of $p<0.05$. Number, percentage, mean, and standard deviation were used to evaluate the data. Chi-square test was used for the groups by giving frequency distributions of categorical data. Student's t-test was used for normally distributed groups to compare the measurements of two groups in a given variable. To compare multiple groups, Tukey's and Tamhane's T2 tests were performed with oneway analysis of variance test. Spearman's rank correlation analysis was used to determine the relationship between numerical variables. In the survival analysis, Kaplan-Meier test log rank (Mantel-Cox) analysis was performed. Receiver operating characteristic (ROC) curve analysis was performed to determine the cut-off values.

\section{RESULTS}

All of these 50 patients, 8 had grade II diffuse astrocytoma IDH mutant, 3 had grade III anaplastic astrocytoma IDH mutant, 2 had grade III anaplastic astrocytoma IDH wild-type, 3 had grade II oligodendroglioma IDH mutant, 1 had grade III anaplastic oligodendroglioma IDH mutant, 1 had grade III anaplastic oligodendroglioma IDH wild-type, 7 had grade IV glioblastomas IDH mutant, 25 had grade IV glioblastomas IDH wild-type. All patients were IDH1 stained; 22 of them were IDH mutant (44\%) and 28 were IDH wild-type (56\%).
$\mathrm{IDH}-$ mutant tumors were commonly located in the frontal lobe (54.3\%), whereas IDH wild-type tumors were commonly located in the parietal and temporal lobes (69.8\%).

A significant difference in PWI rCBV and DWI ADC coefficients was seen among grade II, III, and IV tumor groups $(p<0.001)$. PWI rCBV cut-off value for grade IV tumors was 2.05 (90\% sensitivity, $78 \%$ specificity). There was no significant difference among grade II, III, and IV tumor groups for Cho/NAA, but Cho/ NAA ratio increased as the tumor grade increased $(p=0.055$; Table I). There was no difference in the $\mathrm{Cho/Cr}$ ratio among grade II, III, and IV tumor groups.

There was a significant difference in PWI rCBV between the IDH mutant and wild type $(\mathrm{p}<0.001)$. The mean value of PWI rCBV in IDH-mutant was $1.92 \pm 0.75$, whereas rCBV in IDH wild type was $3.07 \pm 0.99$. In this study, PWI rCBV cut-off value of IDH mutant-wild type was 2.15 (85\% sensitivity, $64 \%$ specificity).

There was no difference between the IDH mutant and wild type in terms of Cho/Cr, Cho/NAA, and DWI ADC coefficient ( $p>0.05)$. The mean PWI rCBV value was $1.40 \pm 0.41$ in lowgrade glioma (LGG) and $2.89 \pm 0.94$ in high-grade glioma (HGG). PWI rCBV was significantly different in terms of LGG and HGG $(p<0.001)$. The DWI ADC coefficient was statistically significant in terms of $L G G$ and $H G G$ discrimination $(p<0.001)$. There was no significant difference in $\mathrm{Cho/Cr}$ and $\mathrm{Cho/NAA}$ ratios between LGG and HGG ( $p>0.05$; Table II).

Table I: Comparison of Grade II, III, and IV Tumors with aMRI Findings and Ki-67 Proliferation Value

\begin{tabular}{|c|c|c|c|c|c|c|c|c|c|c|}
\hline & & $\mathrm{n}$ & Mean & $\begin{array}{l}\text { Standard } \\
\text { deviation }\end{array}$ & Minimum & Maximum & $\begin{array}{c}p \\
\text { (ANOVA) }\end{array}$ & & ost-Hoc T & \\
\hline \multirow{4}{*}{ PWI (rCBV) } & Grade II & 11 & 1.40 & 0.41 & 1.10 & 2.50 & \multirow{4}{*}{$<0.001$} & \multirow{2}{*}{ Grade III } & Grade II & 0.24 \\
\hline & Grade III & 7 & 2.03 & 0.54 & 1.30 & 3.00 & & & Grade IV & 0.01 \\
\hline & Grade IV & 32 & 3.08 & 0.91 & 1.80 & 6.00 & & \multirow{2}{*}{ Grade IV } & Grade II & $<0.001$ \\
\hline & Total & 50 & 2.57 & 1.05 & 1.10 & 6.00 & & & Grade III & 0.01 \\
\hline \multirow{4}{*}{ Cho/Cre } & Grade II & 11 & 3.02 & 1.31 & 2.00 & 6.00 & \multirow{4}{*}{0.268} & \multirow{2}{*}{ Grade II } & Grade III & 0.98 \\
\hline & Grade III & 7 & 3.17 & 1.48 & 1.50 & 5.20 & & & Grade IV & 0.31 \\
\hline & Grade IV & 32 & 3.88 & 1.78 & 1.50 & 9.00 & & \multirow{2}{*}{ Grade III } & Gradell & 0.98 \\
\hline & Total & 50 & 3.59 & 1.67 & 1.50 & 9.00 & & & Grade IV & 0.57 \\
\hline \multirow{4}{*}{ Cho/NAA } & Grade II & 11 & 5.37 & 4.20 & 2.40 & 14.00 & \multirow{4}{*}{0.055} & \multirow{2}{*}{ Grade IV } & Grade II & 0.14 \\
\hline & Grade III & 7 & 4.89 & 3.71 & 2.10 & 12.00 & & & Grade III & 0.14 \\
\hline & Grade IV & 32 & 8.02 & 3.83 & 1.90 & 18.00 & & \multirow{2}{*}{ Grade II } & GradellI & 0.96 \\
\hline & Total & 50 & 7.00 & 4.06 & 1.90 & 18.00 & & & Grade IV & 0.14 \\
\hline \multirow{4}{*}{$\begin{array}{l}\text { DWI ADC } \\
\left({ }^{*} 10^{-3} \times 10^{-3}\right. \\
\left.\mathrm{mm}^{2} / \mathrm{sn}\right)\end{array}$} & Grade II & 11 & 107.36 & 44.52 & 56 & 177.00 & \multirow{4}{*}{$<0.001$} & \multirow{2}{*}{ Grade III } & Grade II & $<0.001$ \\
\hline & Grade III & 7 & 193.43 & 23.56 & 159 & 228.00 & & & Grade IV & 1.00 \\
\hline & Grade IV & 32 & 191.81 & 55.49 & 122 & 350.00 & & \multirow{2}{*}{ Grade IV } & Grade II & $<0.001$ \\
\hline & Total & 50 & 173.46 & 60.65 & 56 & 350.00 & & & Grade III & 1.00 \\
\hline
\end{tabular}


Gezer B. et al: Immunogenetic Properties of Glial Tumors

Table II: Comparison of LGG (Low-Grade Glioma) and HGG (High-Grade Glioma) in Terms of aMRI Findings

\begin{tabular}{|c|c|c|c|c|c|c|c|c|c|c|}
\hline & & $\mathbf{n}$ & Mean & $\begin{array}{l}\text { Standard } \\
\text { deviation }\end{array}$ & Minimum & Maximum & $\begin{array}{c}p \\
\text { (ANOVA) }\end{array}$ & $\mathbf{p}(\mathbf{P}$ & st-Hoc Tes & \\
\hline \multirow{4}{*}{ PWI (rCBV) } & Grade II & 11 & 1.40 & 0.41 & 1.10 & 2.50 & \multirow{4}{*}{$<0.001$} & \multirow{2}{*}{ Grade III } & Grade II & 0.24 \\
\hline & Grade III & 7 & 2.03 & 0.54 & 1.30 & 3.00 & & & Grade IV & 0.01 \\
\hline & Grade IV & 32 & 3.08 & 0.91 & 1.80 & 6.00 & & \multirow{2}{*}{ Grade IV } & Grade II & $<0.001$ \\
\hline & Total & 50 & 2.57 & 1.05 & 1.10 & 6.00 & & & Grade III & 0.01 \\
\hline \multirow{4}{*}{ Cho/Cre } & Grade II & 11 & 3.02 & 1.31 & 2.00 & 6.00 & \multirow{4}{*}{0.268} & \multirow{2}{*}{ Grade II } & Grade III & 0.98 \\
\hline & Grade III & 7 & 3.17 & 1.48 & 1.50 & 5.20 & & & Grade IV & 0.31 \\
\hline & Grade IV & 32 & 3.88 & 1.78 & 1.50 & 9.00 & & \multirow{2}{*}{ Grade III } & Grade II & 0.98 \\
\hline & Total & 50 & 3.59 & 1.67 & 1.50 & 9.00 & & & Grade IV & 0.57 \\
\hline \multirow{4}{*}{ Cho/NAA } & Grade II & 11 & 5.37 & 4.20 & 2.40 & 14.00 & \multirow{4}{*}{0.055} & \multirow{2}{*}{ Grade IV } & Grade II & 0.14 \\
\hline & Grade III & 7 & 4.89 & 3.71 & 2.10 & 12.00 & & & Grade III & 0.14 \\
\hline & Grade IV & 32 & 8.02 & 3.83 & 1.90 & 18.00 & & \multirow{2}{*}{ Grade II } & Grade III & 0.96 \\
\hline & Total & 50 & 7.00 & 4.06 & 1.90 & 18.00 & & & Grade IV & 0.14 \\
\hline \multirow{4}{*}{$\begin{array}{l}\text { DWI ADC } \\
\left({ }^{\star} 10^{-3} \times 10^{-3}\right. \\
\left.\mathrm{mm}^{2} / \mathrm{sn}\right)\end{array}$} & Grade II & 11 & 107.36 & 44.52 & 56 & 177.00 & \multirow{4}{*}{$<0.001$} & \multirow{2}{*}{ Grade III } & Grade II & $<0.001$ \\
\hline & Grade III & 7 & 193.43 & 23.56 & 159 & 228.00 & & & Grade IV & 1.00 \\
\hline & Grade IV & 32 & 191.81 & 55.49 & 122 & 350.00 & & \multirow{2}{*}{ Grade IV } & Grade II & $<0.001$ \\
\hline & Total & 50 & 173.46 & 60.65 & 56 & 350.00 & & & Grade III & 1.00 \\
\hline
\end{tabular}

\section{DISCUSSION}

The accuracy of cMRI in grading glial tumors is $55 \%-85 \%$ $(21,38)$. Parameters such as heterogeneity, contrast enhancement, necrosis, peripheral edema, and cystic content are used for grading; however, recent studies have shown that tumor grade does not always depend on these parameters (36). Tumor grade is not always uniform; additionally, heterogeneous tumors may have lower-grade areas $(25,29,32)$. Therefore, in biopsies, tumors may be diagnosed as low grade. However, imaging methods evaluate the whole lesion. In patients with surgical or biopsy risk due to the location of the lesion, radiological imaging may be the only option for grading (18). Hence, the limitation of $\mathrm{cMRI}$ in grading is an important concern (3). MRS, which makes it possible to evaluate the biochemistry and metabolism of the lesion; MR perfusion, which gives information about microcirculation; and MR diffusion, which gives information about cellularity, play an important role in tumor grading.

Carrillo et al. suggested that imaging properties can be used to estimate the IDH1 mutation with $97.5 \%$ accuracy (7). In this study, no contrast was noted in all IDH1 mutant tumors, and most of the IDH1 tumors had frontal lobe involvement. In our study IDH wild-type tumors were statistically significant in contrast, whereas IDH-mutant tumors were statistically significant without contrast $(\mathrm{p}<0.001)$. IDH-mutant tumors were commonly located in the frontal lobe (54.3\%); IDH wild-type tumors were commonly located in the parietal and temporal lobes (69.8\%), which is compatible with the literature.
Several studies have shown differences in aMRI measurements between IDH wild-type and IDH-mutant glial tumors $(8,16,24,32,34)$. Probably, the observed differences in the rCBV and ADC, Cho/Cr, and Cho/NAA ratios reflect the known differences between the appearance of tumor cell morphology and vascular biology (30). Despite these interesting observations, there is a critical gap in our current understanding of the differences between aMRI values, histological and genetic subtypes of tumors, and differences between glial tumors of the same subtype. We have hypothesized that a combination of PWI, DWI, and MRS measurements distinguishes better criteria based on the genetic characteristics of tumors (26).

The role of MRS in tumor grading has been investigated in numerous studies. Segebarth et al. reported that the Cho/ NAA and Cho/Cr ratios were reliable in glioma grading (31). NAA peak loss is observed in the tumor tissue due to loss of normal neurons. In addition to NAA peak loss in brain tumors, an increase in choline levels is expected to increase in Cho/ NAA ratio (19). Yang et al. found significant differences in Cho/ Cr ratio in low-high-grade separation in theirstudies involving 17 gliomas (37). Cho values correlate with tumor cellularity $(12,17,19)$. However, cellularity may not always accurately reflect the grade; the more important parameters in grading are mitotic activity, necrosis, and vascular proliferation (37). Therefore, the Cho/Cr ratio may not always be compatible with grade. In this study, there was no significant difference in the Cho/Cr and Cho/NAA ratios in the grading of glial tumors. There was also no significant difference between these ratios 
in whether the tumor is IDH wild or mutant. Present study results showed that MRS might not be used as a diagnostic tool for predicting immunogenetic properties of glial tumors.

The role of DWI ADC in terms of tumor grading (low or high) has been documented well in previous studies. However, its predictabilty inimmunogenetic properties is lacking. Diehn et al. found no significant difference in ADC in nonnecrotic tumor areas between patients with IDH wild-type and IDH-mutant tumors (13). Noguchi et al. also did not find a significant difference between IDH wild-type and IDH-mutant tumors in their study. The DWI ADC results were statistically significant in terms of LGG and HGG discrimination. However, there was no significant difference between IDH wild-type and IDH-mutant tumors found in this study (28). This results are compatible with the literature.

MR perfusion imaging provides indirect information about tumor angiogenesis by showing cerebral microcirculation $(3,6,23)$. The advantage of this technique is that it can deliver this information even without the blood-brain barrier damage required for contrast-enhanced MRI. Additionally, rCBV makes it possible to quantify the increased brain tumor vascularity. Because tumor vascularity is an important part of histological grading, this data is valuable for radiological grading $(4,33)$. Studies have shown that rCBV values may be useful in glioma grading. In the study of Law et al., LGG and HGG rCBV values were 2.14 and 5.19, respectively (22). Aronen et al. found that HGG often increased areas of blood volume and decreased but LGG was more homogeneous in this aspect (3). Yang et al. reported that high cellularity may limit vascularity and that rCBV values may be higher at tumor borders (37). Although there is no consensus threshold in the literature, the reported values are generally in the range of $1.75-2(1,14)$. A significant difference in rCBV values between the grades $(p<0.001)$ was found in this study. The cut-off value of rCBV for grade IV tumors was 2.05 (90\% sensitivity, 78\% specificity). While this difference was present between LGG and HGG, no significant difference between grade III and IV $(p<0.001)$ was found. In the presented study we also evaluated the value of PWI rCBV values regarding types of IDH. There was a statistically significant difference in the PWI rCBV value between the IDH mutant and wild type $(\mathrm{p}<0.001)$. The mean $\mathrm{rCBV}$ ratio in patients with IDH-mutant tumors was $1.92 \pm 0.75$, whereas that in those with IDH wild type was $3.07 \pm 0.99$. The PWI rCBV cut-off value in IDH mutant-wild type was $2.15(85 \%$ sensitivity, $64 \%$ specificity). In this study we have determined a cut-off value to discriminate types of IDH for the first time in literature.

\section{CONCLUSION}

PWI rCBV value is the most important prognostic value of an MRI used in tumor grading. There was no correlation between $\mathrm{Cho} / \mathrm{Cr}$ and Cho/NAA ratios in tumor grading. However, Cho/ NAA values were significant when evaluated with PWI rCBV and DWI ADC coefficients.

Furthermore, PWI rCBV values could be significant in the distinction of these two types. This is the only study showing a
PWI rCBV cut-off value of 2.15. DWI and MRS values showed no significant relationship between IDH mutant and wild type.

\section{REFERENCES}

1. Al-Okaili RN, Krejza J, Woo JH: Intraaxial brain masses: MR imagingbased diagnostic strategy-initial experience. Radiology 243:539-550, 2007

2. Anselmi M, Catalucci A, Felli V, Vellucci V: Diagnostic accuracy of proton magnetic resonance spectroscopy and perfusionweighted imaging in brain gliomas follow-up: A single institutional experience. Neuroradial J 30(3):240-252, 2017

3. Aronen HJ, Gazit IE, Louis DN, Buchbinder BR, Pardo FS, Weisskoff RM, Harsh GR, Cosgrove GR, Halpern EF, Hochberg $\mathrm{FH}$ : Cerebral blood volume maps of gliomas: Comparison with tumor grade and histologic findings. Radiology 191:4151, 1994

4. Batra A, Tripathi RP, Singh AK: Perfusion magnetic resonance imaging and magnetic resonance spectroscopy of cerebral gliomas showing imperceptible contrast enhancement on conventional magnetic resonance imaging. Australas Radiol 48(3):324-332, 2004

5. Boxerman JL, Schmainda KM, Weisskoff RM: Relative cerebral blood volume maps corrected for contrast agent extravasation significantly correlate with glioma tumor grade, whereas uncorrected maps do not. AJNR Am J Neuroradiol 27:859-867, 2006

6. Cai J, Zhang C, Zhang W, Wang G, Yao K, Wang Z, Li G, Qian Z, Li Y, Jiang T, Jiang C: ATRX, IDH1-R132H and Ki-67 immunohistochemistry as a classification scheme for astrocytictumors. Oncoscience 3(7-8):258-265, 2016

7. Carrillo JA, Lai A, Nghiemphu PL, Kim HJ, Phillips HS, Kharbanda S, Moftakhar P, Lalaezari S, Yong W, Ellingson BM, Cloughesy TF, Pope WB: Relationship between tumor enhancement, edema, IDH1 mutational status, MGMT promoter methylation, and survival in glioblastoma. AJNR Am J Neuroradiol 33:1349-1355, 2012

8. Cha S, Tihan T, Crawford F, Fischbein NJ, Chang S, Bollen A, Nelson SJ, Prados M, Berger MS, Dillon WP: Differentiation of low-grade oligodendrogliomas from low-grade astrocytomas by using quantitative blood-volume measurements derived from dynamic susceptibility contrast-enhanced MR imaging. AJNR Am J Neuroradiol 26:266-273, 2005

9. Chawla S, Krejza J, Vossough A, Zhang Y, Kapoor GS, Wang S, O'Rourke DM, Melhem ER, Poptani H: Differentiation between oligodendroglioma genotypes using dynamic susceptibility contrast perfusion-weighted imaging and proton MR spectroscopy. AJNR Am J Neuroradiol 34:15421549, 2013

10. Chen L, Liu M, Bao J, Xia Y, Zhang J, Zhang L, Huang X, Wang $\mathrm{J}$ : The correlation between apparent diffusion coefficient and tumor cellularity in patients: A meta-analysis. PLoS ONE 8:e79008, 2013

11. Chen WJ, He DS, Tang RX, Ren FH, Chen G: Ki-67 is a valuable prognostic factor in gliomas: Evidence from a systematic review and meta-analysis. Asian Pac J Cancer Prev 16 (2):411-420, 2015 
12. Croteau D, Scarpace L, Hearshen D: Correlation between magnetic resonance spectroscopy imaging and image-guided biopsies: Semiquantitative and qualitative histopathological analyses of patients with untreated glioma. Neurosurgery 49(4): 823-829, 2001

13. Diehn M, Nardini C, Wang DS: Identification of noninvasive imaging surrogates for brain tumor gene-expression modules. Proc Natl Acad Sci USA 105:5213-5218, 2008

14. Ellingson BM, Malkin MG, Rand SD, Connelly JM, Quinsey C, LaViolette PS, Bedekar DP, Schmainda KM: Validation of functional diffusion maps (fDMs) as a biomarker for human glioma cellularity. J Magn Reson Imaging 31:538-548, 2010

15. Emblem KE, Nedregaard B, Nome T: Glioma grading by using histogram analysis of blood volume heterogeneity from MRderived cerebral blood volume maps. Radiology 247:808-817, 2008

16. Fellah S, Caudal D, De Paula AM, Dory-Lautrec P, FigarellaBranger D, Chinot O, Metellus P, Cozzone PJ, Confort-Gouny S, Ghattas B, Callot V, Girard N: Multimodal MR imaging (diffusion, perfusion, and spectroscopy): Is it possible to distinguish oligodendroglial tumor grade and $1 p / 19 q$ codeletion in the pretherapeutic diagnosis? AJNR Am J Neuroradiol 34:1326-1333, 2013

17. Gupta RK, Cloughesy TF, Sinha U: Relationships between choline magnetic resonance spectroscopy, apparent diffusion coefficient and quantitative histopathology in human glioma. J Neurooncol 50(3):215-226, 2000

18. Hakyemez B, Erdogan C, Ercan I: High-grade and low-grade gliomas: Differentiation by using perfusion MR imaging. Clin Radiol 60:493-502, 2005

19. Horská A, Barker PB: Imaging of brain tumors: MR spectroscopy and metabolic imaging. Neuroimaging Clin $\mathrm{N}$ Am 20(3):293-310, 2010

20. Ichimura K, Pearson DM, Kocialkowski S, Backlund LM, Chan R, Jones DT, Collins VP: IDH1 mutations are present in the majority of common adult gliomas but rare in primary glioblastomas. Neuro Oncol 11:341-347, 2009

21. Knopp EA, Cha S, Johnson G, Mazumdar A, Golfinos JG, Zagzag D, Miller DC, Kelly PJ, Kricheff II: Glial neoplasms: Dynamic contrast-enhanced T2- weighted MR imaging. Radiology 211:791-798, 1999

22. Law M, Yang S, Wang H, Babb JS, Johnson G, Cha S, Knopp EA, Zagzag D: Glioma grading: Sensitivity, specificity, and predictive values of perfusion MR imaging and proton MR spectroscopic imaging compared with conventional MR imaging. AJNR Am J Neuroradiol 24:1989-1998, 2003

23. Lee SJ, Kim JH, Kim YM, Wog K: Perfusion MR imaging in gliomas: Comparison with histologic tumour grade. Korean $\mathrm{J}$ Radiol 2:1-7, 2001

24. Lev MH, Ozsunar Y, Henson JW, Rasheed AA, Barest GD, Harsh GRt, Fitzek MM, Chiocca EA, Rabinov JD, Csavoy AN, Rosen BR, Hochberg FH, Schaefer PW, Gonzalez RG: Glial tumor grading and outcome prediction using dynamic spinecho MR susceptibility mapping compared with conventional contrast-enhanced MR: Confounding effect of elevated rCBV of oligodendrogliomas. AJNR Am J Neuroradiol 25:214-221, 2004
25. Li C, Ai B, Li Y, Qi H, Wu L: Susceptibility-weighted imaging in grading brain astrocytomas. Eur J Radiol 75:e81-e85, 2010

26. Louis DN, Perry A, Reifenberger G, von Deimling A, FigarellaBranger D, Cavenee WK, Ohgaki H, Wiestler OD, Kleihues P, Ellison DW: The 2016 World Health Organization classification of tumors of the central nervous system: A summary. Acta Neuropathol 131:803-820, 2016

27. Nobusawa S, Watanabe T, Kleihues $P$, Ohgaki H: IDH1 mutations as molecular signature and predictive factor of secondary glioblastomas. Clin Cancer Res 15:6002-6007, 2009

28. Noguchi T, Yoshiura T, Hiwatashi A, Togao O, Yamashita K, Nagao E, Shono T, Mizoguchi M, Nagata S, Sasaki T, Suzuki SO, Iwaki T, Kobayashi K, Mihara F, Honda H: Perfusion imaging of brain tumors using arterial spin-labeling: Correlation with histopathologic vascular density. AJNR Am J Neuroradiol 29(4):688-693, 2008

29. Pinker K, Noebauer-Huhmann IM, Stavrou I: High-resolution contrastenhanced, susceptibility-weighted MR imaging at 3T in patients with brain tumors: Correlation with positronemission tomography and histopathologic findings. AJNR Am J Neuroradiol 28:1280-1286, 2007

30. Schiffer D, Bosone I, Dutto A, Di Vito N, Chio A: The prognostic role of vessel productive changes and vessel density in oligodendroglioma. J Neurooncol 44:99-107, 1999

31. Segebarth CM, Baleriaux DF, Luyten PR, den Hollander JA: Detection of metabolic heterogeneity of human intracranial tumors in vivo by $1 \mathrm{H}$ NMR spectroscopic imaging. Magn Reson Med 13(1):62-76, 1990

32. Sentürk S, Oguz K, Cila A: Dynamic contrastenhancedsusceptibilityweighted perfusion imaging of intracranial tumors: A study using a 3T MR scanner. Diagn Interv Radiol 15:3-12, 2009

33. Spampinato MW, Smith JK, Kwock L, Ewend M, Grimme JD, Camacho DL, Castillo M: Cerebral blood volume measurements and proton MR spectroscopy in grading of oligodendroglial tumors. AJR Am J Roentgenol 188(1):204212, 2007

34. Tan W, Xiong J, Huang W, Wu J, Zhan S, Geng D: Noninvasively detecting Isocitrate dehydrogenase 1 gene status in astrocytoma by dynamic susceptibility contrast MRI. J MagnReson Imaging 45:492-499, 2016

35. Watanabe T, Nobusawa S, Kleihues P, Ohgaki H: IDH1 mutations are early events in the development of astrocytomas and oligodendrogliomas. Am J Pathol 174:1149-1153, 2009

36. Yan H, Parsons DW, Jin G, McLendon R, Rasheed BA, Yuan W, Kos I, Batinic-Haberle I, Jones S, Riggins GJ, Friedman H, Friedman A, Reardon D, Herndon J, Kinzler KW, Velculescu VE, Vogelstein B, Bigner DD: IDH1 and IDH2 mutations in gliomas. N Engl J Med 360:765-773, 2009

37. Yang D, Korogi Y, Sugahara T: Cerebral gliomas: Prospective comparison of multivoxel 2D chemical-shift imaging proton MR spectroscopy, echoplanar perfusion and diffusionweighted MRI. Neuroradiology 44:656-666, 2002

38. Zonari P, Baraldi P, Crisi G: Multimodal MRI in the characterization of glialneoplasms: The combined role of single-voxel MR spectroscopy, diffusion imaging and echo-planar perfusion imaging. Neuroradiology 49(10):795-803, 2007 